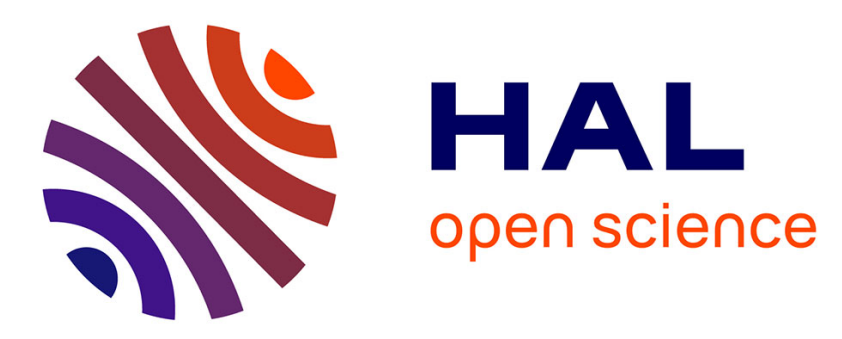

\title{
Analysis of index modulation in microembolic Doppler signals part I: radiation force as a new hypothesis-simulations
}

Jean-Marc Girault, Denis Kouamé, Sébastien Ménigot, Grégory Souchon, François Tranquart

\section{To cite this version:}

Jean-Marc Girault, Denis Kouamé, Sébastien Ménigot, Grégory Souchon, François Tranquart. Analysis of index modulation in microembolic Doppler signals part I: radiation force as a new hypothesis-simulations. Ultrasound in Medicine \& Biology, 2011, 37 (1), pp.87-101. 10.1016/j.ultrasmedbio.2010.09.006 . hal-00658788

\section{HAL Id: hal-00658788 \\ https://hal.science/hal-00658788}

Submitted on 11 Jan 2012

HAL is a multi-disciplinary open access archive for the deposit and dissemination of scientific research documents, whether they are published or not. The documents may come from teaching and research institutions in France or abroad, or from public or private research centers.
L'archive ouverte pluridisciplinaire HAL, est destinée au dépôt et à la diffusion de documents scientifiques de niveau recherche, publiés ou non, émanant des établissements d'enseignement et de recherche français ou étrangers, des laboratoires publics ou privés. 


\title{
Analysis of Index Modulation in microembolic Doppler signals Part I: Radiation Force as a new hypothesis - simulations
}

\author{
Jean-Marc Girault, ${ }^{\text {a }}$ Denis Kouamé, b Sébastien Ménigot, ${ }^{a}$ \\ Grégory Souchon, ${ }^{\mathrm{a}}$ François Tranquart ${ }^{\mathrm{c}}$ \\ "Université François Rabelais de Tours, UMRS "Imaging and Brain" U930 and CNRS ERL \\ 3106, Tours, France. \\ ${ }^{\mathrm{b}}$ Université PAUL Sabatier de Toulouse 3, IRIT UMR 5505, France \\ ${ }^{\mathrm{c}}$ Bracco, Switzerland
}

Corresponding Author: Jean-Marc Girault

Inserm U930 - CNRS ERL 3106 - Équipe 5

UFR de Médecine

10 boulevard Tonnellé, BP3223

F-37032 TOURS CEDEX 1

Email: jean-marc.girault@univ-tours.fr

Telephone: +33 (0)2 47366221

Elsevier

Ultrasound in Medicine \& Biology, Volume 37, Issue 1, January 2011, Pages 87-101

DOI : 10.1016/j.ultrasmedbio.2010.09.006

http://www.sciencedirect.com/science/article/pii/S0301562910005028 


\begin{abstract}
The purpose of this study was to reveal the cause of frequency modulation (FM) present in microembolic Doppler ultrasound signals. This novel explanation should help the development of sensitive microembolus discrimination techniques. We suggest that the frequency modulation detected is due to the ultrasonic radiation force (URF) acting directly on microemboli. The frequency modulation and the imposed displacement were calculated using a numerical dynamic model. By setting simulation parameters with practical values, it was possible to reproduce most microembolic frequency modulation signatures (FMS). The most interesting findings in this study were that: 1) the ultrasound radiation force acting on a gaseous microembolus and its corresponding cumulative displacement were far higher than those obtained for a solid microembolus, and that is encouraging for discrimination purposes, and 2) the calculated frequency modulation indices $(\mathrm{FMI})(\approx 20 \mathrm{kHz})$ were in good agreement with literature results. By taking into account the ultrasound radiation force, the flow pulsatility, the beam-to-flow angle and both the velocity and the US beam profiles, it was possible to explain all erratic FM signatures of a microbubble. Finally, by measuring FMI from simulated Doppler signals and by using a constant threshold of $1 \mathrm{KHz}$, it was possible to discriminate gaseous from solid microemboli with ease.
\end{abstract}

Key words: Discrimination, Microemboli, ultrasound radiation force, frequency modulation signature. 
2 Cerebral embolisms represent a significant proportion of all ischemic strokes in western 3 countries, and identification of types of microemboli (gaseous or solid) and size may be 4 important for diagnosis and subsequent treatment. The Transcranial Doppler (TCD) sys5 tem is the most commonly used method for microembolus detection, and time-frequency 6 representation provides valuable indications (frequency modulation) of the nature and 7 size of a microembolus.

Many reports have been published concerning the differentiation of gaseous and particulate matter ((Rusell et al., 1991; Markus and Brown, 1993; Georgiadis et al., 1994; Smith et al., 1997, 1998; Rusell and Brucher, 2002)). Some of them have focused on the measurement of microembolus velocity or duration (Markus and Brown, 1993; Georgiadis et al., 1994; Rusell and Brucher, 2002), and others on the measurement of the microembolic signal (MES) intensity (Markus and Brown, 1993; Smith et al., 1997). In practice, when a TCD system detects high intensity transient signals (HITS) many interpretations are possible based on the scattering theory: they may be due to a small gaseous microembolus (a few microns) such as from a prosthetic heart valve, a small formed element (tens of microns) such as from prosthetic a heart valve or an arterial lesion with ulceration, a large bubble such as from air trapped in the vasculature during surgery, or a large piece of clot such as from atrial fibrillation. Hence, most of the parameters used for discrimination implemented in on- or off-line systems are based on the linearity of the backscattered Doppler signal. The most popular are embolic duration (ED) (Markus and Brown, 1993; Smith et al., 1997), embolic velocity (EV) (Smith et al., 1997), embolus to blood ratio (EBR) (Markus and Brown, 1993; Smith et al., 1997; Rusell and Brucher, 2002), signal to error ratio (SER) (Devuyst et al., 2000), frequency band and symmetry of the HITS signature (Devuyst et al., 2001) and sample volume length (SVL) (Smith et al., 1998). However, the parameter which seems to us the most interesting and relevant is the frequency modulation index (FMI) (Smith et al., 1997). This parameter, which measures the slope 
intensity of the microembolic frequency modulation signature (FMS), is important since it is based on measuring the trajectory modification of microembolus circulation in the blood stream. The physical phenomenon which underlies this "additional-displacement" has not been clearly identified and we believe that discovering the correct explanation should lead researchers to propose more sensitive discrimination techniques.

The starting point of our study was the article written by Smith et al. (1997). The most remarkable finding of this study was that known solid microemboli never appear to give rise to Doppler MES with rapidly changing frequency modulations (FM). In our opinion this study is one of the most interesting for two reasons. First because it is based on an extensive clinical study and second because several valuable explanations are proposed. The clinical studies of Smith et al. (1997) and those referenced in the same article showed that the Doppler MES from patients with a prosthetic heart valve present a high frequency modulation index (FMI) whereas the FMI is low in patients with atherosclerotic plaques. An in vitro study reported by Souchon et al. (2005) confirmed that gaseous microemboli produced higher FMI than those produced by solid microemboli. Smith et al. (1997) proposed some new and valuable hypotheses such as artery curvature, helical flow, harmonic generation and phase cancelation to explain the different types of FM. Furthermore, Smith et al. (1997) proposed three distinct types of FM: those with no modulation (type I), those with gradual FM observed over the majority of the signal duration (type II) and those with a rapid change evident in only a small percentage of the entire signal duration (type III). The most surprising type III FM signatures observed in the timefrequency domain resemble a "V" shape or a reversed "V" shape. We propose here a valid explanation for these strange signatures.

The aim of the present study was to extend the results of Smith et al. (1997). To explain the three types of FM, we hypothesized that the major physical phenomenon involved in the modification of the microembolus trajectory is the ultrasound radiation force (URF) and therefore studied many aspects. Many factors were studied to prove that the URF explains these strange FMS. First, the necessary background physics are reviewed, and 
the dynamics of a microembolus crossing an ultrasound beam are examined. A numerical simulation based on different models is then introduced to calculate the theoretical values of both the URF and the microdisplacement. Finally, different types of FMS calculated from simulated Doppler microembolic signals are discussed and several valuable explanations are proposed.

\section{Materials and Methods}

Theoretical models and equations which explain how a microembolus in a circulating fluid can be displaced by a radiation force are presented in this section. After recalling Newton's law to describe the different movements, the different forces acting on the circulating microemboli (see Fig.1) are taken into consideration, and the drag force and the radiation force are introduced. Finally, a numerical model is discussed which predicts the modification of the microembolus trajectory .

\section{Newton's law}

The "additional displacement" (or rather the "additional acceleration") of a microembolus due to the radiation force is governed by Newton's law. This is an additional acceleration to the natural acceleration induced by the spatiotemporal blood flow in which the microembolus is circulating. Newton's law is written:

$$
\sum \vec{F}=\overrightarrow{F_{U S}}+\overrightarrow{F_{D G}}=\tilde{m} \vec{\gamma}
$$

where $\tilde{m}$ corresponds to the mass of the microembolus and $\gamma$ is the acceleration. In the case of gaseous microemboli, the effective mass corresponds to the fluid mass displaced, the effective mass here being set at 0.5 times the mass surrounding the displaced fluid (Leighton, 1994). The forces acting on the micro embolus are the drag force $\overrightarrow{F_{D G}}$ and the radiation force $\overrightarrow{F_{U S}}$ (gravity being disregarded). As can be seen in the following section, 
95

the drag force is closely related to the properties of the circulating fluid while the radiation force is principally related to the US field.

Drag force

Drag force occurs when there is a relative velocity between a particle and the viscous fluid in which the particle is immersed. When a microembolus is subjected to the radiation force, the latter accelerates according to Newton's law. The acceleration induces a nonnegligible relative velocity between the microembolus and the fluid, which induces a drag force.

For a Newtonian fluid, Benocci et al. (1986) (in the case of a gas-droplet in industrial flow) and Tortoli et al. (2000) (in the case of contrast agent circulating in blood) proposed a drag force model acting on a spherical microembolus (with a radius $R_{0}$ ) expressed as follows:

$$
F_{D G}=F_{D G}^{\prime} \frac{C_{D} R e}{24} \rho
$$

where $F_{D G}^{\prime}$ is the drag force in the case of laminar flow, $\rho$ is the density of the host fluid, and $R e$ and $C_{D}$ are the Reynolds number and the drag coefficient, respectively. These parameters are expressed by:

$$
\begin{aligned}
& F_{D G}^{\prime}=\frac{3}{2} \pi R_{0} \eta\left\|V_{f}-V_{b}\right\|, \\
& R e=\frac{\left\|V_{f}-V_{b}\right\|}{\eta}, \\
& C_{D}=\frac{24}{R e}+\frac{6}{1+\sqrt{R e}}+0.4,
\end{aligned}
$$

where $\eta$ is the fluid viscosity, $R_{0}$ is the radius of the microembolus, and $V_{f}$ and $V_{b}$ are the fluid and microembolus velocities, respectively.

We emphasize that this force is strongly dependent on the spatial velocity distribution 
(laminar or plug profile) and on the heartbeat (systolic or diastolic phase). The effects of spatial velocity distribution will be discussed later.

\section{Radiation Force}

The acoustic radiation pressure on rigid spheres freely suspended in a fluid was first calculated by King (1934). Yosioka and Kawasima (1955) extended King's results to spheres of finite compressibility suspended in a non-viscous fluid. Doinikov (1996, 1997a,b,c, 1998) extended the Yosioka and Kawasima model by taking thermal and viscous phenomena into account. The latter theory is therefore appropriate to study the radiation force acting on microemboli composed of fat or clots or even an air microbubble in a viscous fluid (e.g. blood).

We examine below simplified models of radiation force acting first on a solid microembolus and then on a gaseous microembolus. These two models, that are valid for microemboli smaller than the wavelength, are derived from a general model which is not presented here.

\section{Radiation force of a solid microembolus}

Assuming a small "solid" microembolus (i.e. soft material such as fat or blood clot) suspended in plasma, the radiation force provided by the simplified Doinikov (1996) model was reformulated as follows:

$$
F_{U S}=\frac{2 \pi P^{2} R_{0}}{\rho c \omega}\left(R_{0} k\right)^{2}\left(\frac{3 \delta_{v}}{R_{0}}\left(\frac{1-\tilde{\rho} / \rho}{1+2 \tilde{\rho} / \rho}\right)^{2}+\frac{\delta_{t}}{R_{0}} \frac{(\nu-1)}{2\left(1+\tilde{\delta}_{t} \kappa /\left(\delta_{t} \tilde{\kappa}\right)\right)}\right)
$$

where $P$ is the pressure level and $\tilde{\rho}$ and $\rho$ are the density of the microembolus and the plasma, respectively. For this application, where the emitting wavelength is much larger than the radius of the microembolus $\left(\left(k R_{0}\right)^{2} \ll 1,\left(\tilde{k} R_{0}\right)^{2} \ll 1\right.$, where $\tilde{k}=\omega / \tilde{c}$ and $k=\omega / c$ are the wave numbers of the microembolus and the plasma, respectively, $\omega$ is the 


$$
\begin{aligned}
& \beta_{v i s}=\frac{\omega}{2}\left(\frac{4 \eta}{\omega \rho R_{0}^{2}}\right), \\
& \beta_{t h}=\frac{\omega}{2}\left(\frac{\omega_{0}^{2}}{\omega^{2}} \frac{3(\tilde{\nu}-1)}{2 R_{0}}\right),
\end{aligned}
$$

$$
\beta_{t o t}=\beta_{a c}+\beta_{v i s}+\beta_{t h},
$$

$$
\beta_{a c}=\frac{\omega}{2}\left(k R_{0}\right),
$$

When $K=1$ the model was equivalent to those proposed by Leighton (1994) and Dayton et al. (1997), and total damping $\beta_{\text {tot }}$ was defined by: 
148

were very similar to those presented by Prosperetti (1977), Asaki and Marston (1994).

The resonance angular frequency $\omega_{0}^{\prime}$ was expressed as follows:

$$
\omega_{0}^{\prime}=\omega_{0} \sqrt{K_{2}},
$$

where $\omega_{0}$ is the resonance angular frequency when the surface tension $\tilde{\sigma}$ is zero. This resonance angular frequency was defined by :

$$
\omega_{0}=\sqrt{\frac{3 \tilde{\nu} \tilde{p}_{0}}{R_{0}^{2} \rho}},
$$

where $\tilde{p_{0}}$ was the hydrostatic pressure and where $\tilde{\nu} \tilde{p_{0}}=\tilde{\rho} \tilde{c}^{2}$. The weighted coefficients presented in eqn (5) are :

$$
\begin{aligned}
& K=\frac{\beta_{a c}\left(K_{0}-1\right)+\beta_{v i s}\left(K_{1}-1\right)+\beta_{t h}\left(K_{2}-1\right)}{\beta_{t o t}}, \\
& K_{0}=2+\frac{\beta_{v i s}+\beta_{t h}}{\beta_{a c}}
\end{aligned}
$$

$$
\begin{aligned}
& K_{1}=\frac{7}{4}-\frac{3}{4}\left(\frac{\omega_{0}^{\prime}}{\omega}\right)^{2}, \\
& K_{2}=\frac{\omega_{0}^{\prime 2}}{\omega_{0}^{2}}=1-\frac{2 \tilde{\sigma}}{3 R_{0} \tilde{\rho} \tilde{c}^{2}} .
\end{aligned}
$$

\section{Microembolus trajectory}

In this section we present the validation of the feasibility of a differentiation method based on radiation force, using the numerical model initially developed by Tortoli et al. (2000) and used by Palanchon et al. (2005) to estimate the displacement of microbubbles crossing an ultrasound beam in an experimental setup.

The orthogonal projections of the radiation and drag forces on the $\vec{r}$ and $\vec{z}$ axis (see 
157

162

$167 \quad\left\{\begin{array}{l}\Delta_{r}(n+1)=V_{b r}(n) \cdot d t+\Delta_{r}(n), \\ \Delta_{z}(n+1)=V_{b z}(n) \cdot d t+\Delta_{z}(n) .\end{array}\right.$

Definition of the Lagrangian velocity makes it possible to compute the trajectory of the microembolus in each direction as:

The cumulative displacement was defined by $\Delta_{D}=\sqrt{\Delta_{r}^{2}+\Delta_{z}^{2}}$ and the elementary displacement defined by $\delta_{D}=\sqrt{\delta_{r}^{2}+\delta_{z}^{2}}$ where:

$170 \quad\left\{\begin{array}{l}\delta_{r}(n+1)=\Delta_{r}(n+1)-\Delta_{r}(n), \\ \delta_{z}(n+1)=\Delta_{z}(n+1)-\Delta_{z}(n) .\end{array}\right.$

where $\tilde{m}$ represents the mass of the microembolus. The component of the drag force was computed by splitting the relative velocity $\left\|V_{f}-V_{b}\right\|$ into the two radial and longitudinal components (see Fig.1). As suggested by Tortoli et al. (2000), the Euler one-step method was used to obtain discrete forms of eqn.(11) as:

$$
\left\{\begin{array}{l}
-F_{U S r}+F_{D G r}(n)=\tilde{m} \frac{V_{b r}(n+1)-V_{b r}(n)}{d t}, \\
F_{U S z}-F_{D G z}(n)=\tilde{m} \frac{V_{b z}(n+1)-V_{b z}(n)}{d t} .
\end{array}\right.
$$

The velocity components are thus computed as:

64

$$
\left\{\begin{array}{l}
V_{b r}(n+1)=\frac{-F_{U S r}+F_{D G r}(n)}{\tilde{m}} d t+V_{b r}(n), \\
V_{b z}(n+1)=\frac{F_{U S z}-F_{D G z}(n)}{\tilde{m}} d t+V_{b z}(n) .
\end{array}\right.
$$


Eqns (14), (15) were computed with Matlab (Mathworks, Natick, MA). The various stages that we followed to calculate the trajectories of microemboli were as follows: first we calculated the radiation force acting on the microembolus from the two models presented for gaseous and solid microemboli and then we calculated the trajectories. From these trajectories we evaluated the cumulative displacement $\Delta_{D}$. Note that with regard to the cumulative displacement, $\Delta_{r}$ was always less than $\Delta_{z}$. Indeed, $\Delta_{z}=\Delta_{z}^{R}+\Delta_{z}^{V}$ where $\Delta_{z}^{R}$ corresponded to displacement due only to the radiation force and $\Delta_{z}^{V}$ corresponded to displacement due only to the microembolus velocity, whereas $\Delta_{r}=\Delta_{r}^{R}$. In the case of a plug profile and without ultrasound, $\Delta_{z}^{V}=L$. For a fluid velocity of a few centimeters per second, $\Delta_{D} \approx \Delta_{z}^{V}$, since $\Delta_{z}^{V}$ was masked by $\Delta_{z}^{R}$. Consequently, in order to reveal the influence of the radiation force, we preferred subsequently to calculate $\Delta_{D}^{*}=\sqrt{\Delta_{r}^{2}+\left(\Delta_{z}-L\right)^{2}}$. The trajectory calculation ceased when the microembolus went out of the US beam $\left(\Delta_{z}>L\right.$, where $L$ is the beam width) or out of the artery. Note that the beam width seen by the microembolus was: $L=L_{\text {beam }} / \sin \theta$. Note that as in practice the beam-to-flow angle is near 0 degrees, the beam width seen by the microembolus was much higher. For instance, for $\theta=5$ degrees the beam width was $L \approx 23 \mathrm{~mm}$. In practice the ultrasound beam intersection with the middle cerebral artery (MCA) is wide since the blood flow is mainly parallel to the ultrasound beam axis.

In simulations, an angle of 5 degrees was chosen because it ensures an US beam quasi parallel to the MCA and it guarantees a reasonable computing time (the lower the angle, the longer the computing time).

Since microembolus displacement is dependent on the radiation force, the drag force, the acoustic pressure and the fluid velocity (through eqn (13) to eqn (15)), the shape of the ultrasound beam, the velocity profile in the artery and the flow pulsatility are also factors which significantly influence the phenomenon.

To observe the effects of the shape of the velocity profile we used two configurations for which the US beam was considered as uniform, though the uniform US beam is not a 
realistic hypothesis. However it makes it possible to reveal variations in the velocity alone. In the first configuration, the velocity profile was a plug type, i.e. the flow velocity on the $\vec{z}$ axis was equal regardless of the radial position. In the second, the velocity profile was laminar, with the maximum velocity between 0.1 and $1.0 \mathrm{~m} / \mathrm{s}$ (velocity range usually measured in adult cerebral arteries).

Similarly, to observe the effects of the shape of the ultrasound beam, we used two configurations for which the velocity profile was considered as uniform, this hypothesis making it possible to consider the spatial variations of the pressure level alone. In the first configuration, the spatial distribution of the pressure level at a fixed depth was flat, whereas in the second (which seems more realistic) it was Gaussian.

\section{Discrimination techniques}

By assuming that the URF sufficiently modifies the microbubble trajectory to be observed within the Doppler frequency, it seems natural to propose a discrimination technique based on the detection of Doppler frequency variations. Estimation of the frequency modulation index (FMI) seems to be highly appropriate to detect and quantify these additional accelerations. FMI can be defined by:

$$
F M I(t)=\frac{d F_{D}(t)}{d t}
$$

where $F_{D}=2 f V_{b} \cos \theta / c$ is the Doppler frequency available from a time-frequency representation $\left(V_{b}\right.$ is the microbubble velocity). For instance with $\theta=5$ degrees, $V=0.5 \mathrm{~m} / \mathrm{s}$, $f=2 \mathrm{MHz}, c=1500 \mathrm{~m} / \mathrm{s}, F_{D}=950 \mathrm{~Hz}$. It was more convenient to evaluate the FMI from standard TCD recordings off-line, as real time calculation was too time-consuming for our experimental instrumentation.

Similarly, if a multi-gate TCD system is available (Guidi et al. (2003)), it is also possible to detect and quantify accelerations by means of the position modulation index (PMI). 
For instance, PMI can be defined by:

$$
P M I(t)=\frac{d P_{e}(t)}{d t},
$$

where $P_{e}$ is the microembolus position available from a time-depth representation (see Girault et al. (2010) for example). From evaluated FMI/PMI and by setting an empirical threshold, it is possible to detect and discriminate microemboli.

As the two proposed discrimination techniques are off-line techniques, no particular recommendation are required during the TCD recording whatever the clinical scenario or the brain region explored.

To summarize, we present two models of the radiation force derived from the work of Doinikov (1998). These two models were incorporated into a numerical model which permits calculation of microembolus trajectories in the blood flow. Simulations highlighting the effects of these parameters on the trajectory of the microembolus are presented in the results section. Two parameters, i.e. FMI and PMI, are proposed to detect microembolus "additional acceleration".

\section{Results}

In this section we present the results obtained from simulations of the radiation force (see Figs. 2, 3, 4) and from simulations of the microembolus displacement (see Figs. 5, 6, 7 and Tables 2, 3). In order to validate the different models proposed, we compared simulated data to data derived from the literature (Smith et al., 1997), (Girault et al., 2010). The physical parameters used for these simulations are given in Table 1. Finally, though a microembolus can be a few hundred microns in size, we chose to limit the simulation size range to $1-100$ microns because the proposed models are not valid beyond this range. 
Radiation force

As the ultimate goal was the differentiation of gaseous and formed elements, initially the URFs acting on a microbubble and on a solid particle were compared. To understand fully the ways in which such forces differ, each loss involved in the calculation of the URF was evaluated. Furthermore, as in practice the sizes of microemboli are unknown, it is important to carry out simulations for various sizes.

We present first the simulations using the simplified Doinikov model (eqn. (4)) for a solid particle immersed in blood and then we present the simulations using the simplified Doinikov model (eqn. (5)) for a microbubble.

\section{Ultrasound radiation force acting on a solid particle immersed in blood}

In this part of the study, the losses and then the radiation force were evaluated for various sizes. By comparing $\left(k R_{0}\right)^{3}$ to $\delta_{v} / R_{0}$ and $\delta_{t} / R_{0}$ in eqn. (4), it was possible to evaluate which term would predominate at each $R_{0}$ value. These three terms were compared for different frequencies (1, 2 and $3 \mathrm{MHz}$ ). The simulated results plotted in Fig. 2 show that viscous and thermal effects in eqn. (4) predominated in an area of a few microns $(30 \mu \mathrm{m})$. Above this value, the thermal and the viscous effects were insignificant. For radii of less than 10 microns, the losses (thermal and viscous) were higher for low frequencies than for high frequencies. This means that a high emitting frequency is better than a low frequency. For radii greater than 10 microns, the loss (mainly acoustic) was lower for low frequencies than for high frequencies. This means that a low emitting frequency is better than a high frequency. To ensure a good trade-off in terms of losses, the middle frequency (2 $M H z)$ was selected for the rest of the study.

In terms of the URF, the simulated results plotted in Fig.3 (obtained with a pressure level $P=500 \mathrm{kPa}$ and an emitting frequency $f=2 \mathrm{MHz}$ ) show that the URF acting on a solid particle was weak since its amplitude was below $0.01 \mu \mathrm{N}$. However, as the URF 
increases with size, it is highly probable that for a quasi millimetric solid microembolus the URF is no longer negligible.

\section{Ultrasound radiation force acting on a gaseous microembolus immersed in blood}

In this part of the study, the losses and then the URF were evaluated for various sizes. In order to establish which effects predominated (acoustic, viscous, thermal), we simulated the weighted factor $K$ (see eqn. (10)) and its three components $K_{0}, K_{1}$ and $K_{2}$. We also simulated the damping constants (see eqn. (7)) $\beta_{a c}, \beta_{v i s}$ and $\beta_{t h}$. These simulations were performed for a fixed pressure level $(P=500 \mathrm{kPa})$, a fixed emitting frequency $(f=2 \mathrm{MHz})$ and different radii $(1-100 \mu \mathrm{m})$. Fig. $4 \mathrm{~b}$ shows that:

- thermal damping predominated for a radius $<2 \mu \mathrm{m}$;

- viscous damping predominated for $2 \mu m<R_{0}<5 \mu m$;

- acoustic damping predominated for a radius $>5 \mu \mathrm{m}$.

Fig.4a shows that for $f=2 \mathrm{MHz}$ the global factor $K$ was substantially influenced by the terms attached to $K_{0}$ (acoustic losses) and $K_{1}$ (viscous losses) for $2 \mu m<R_{0}<30 \mu m$.

Fig. 3 shows that the URF acting on a gaseous microembolus was far higher than that acting on a fat microembolus. Moreover, as depicted in this figure, the resonance angular frequency $\omega_{0}^{\prime}$ was slightly shifted from $\omega_{0}$ (which corresponded to the resonance angular frequency in the case of a perfect fluid). Furthermore, due to dissipative effects, the resonance was spread more widely and thus less sharply than that obtained from an ideal fluid.

To summarize, we showed for both microbubbles and fat particles that the losses which limited the level of the URF were principally the thermal effects for radii of less than ten microns and acoustic effects for radii greater than ten microns. We also showed that URF acting on microbubbles were far higher than those acting on formed elements, thus leaving a question regarding microbubble displacement in comparison with that of a fat 
particle.

\section{Displacement}

The main purpose of these simulations (see Fig.5, Table 2, Table 3 and Fig.6) was to propose some novel explanations of the FMS, particularly for the "V" (reversed or unreversed) shape of the time-frequency Doppler microembolic signature. Further significant aims were:

- to provide an order of magnitude of displacements induced by the radiation force and of corresponding measurable parameters (FMI, PMI);

- to improve understanding of the parameters which impact on microembolus displacement and FMI/PMI;

- to show the feasibility of discriminating between fat and gaseous microemboli in plasma (or water) for a wide range of radii;

- to predict and provide an order of magnitude of the cumulative displacement $\Delta_{D}$ of a microembolus circulating in blood or water;

- to verify whether the in vitro measurements reported in the literature are compatible with the simulated values of $\Delta_{D}, F_{D}, F M I$ and $P M I$.

\section{Cumulative displacement}

The aim of this simulation (see Fig.5) was to improve understanding of the evolution of cumulative displacement for different microembolus sizes (gaseous or fat) and to provide an order of magnitude of the displacement. The elementary displacement of the microbubble is an interesting indicator since it is the direct consequence of the radiation force. However, as its value is very low, calculation of the cumulative displacement is preferred.

Fig.5 shows that the cumulative displacements for both gaseous and formed elements 
produced a shape relatively close to the shape of the corresponding URF. However, there were some small differences. It can be seen in Fig.3 that the URF level was the same for gaseous microemboli of 2 microns and 20 microns, while the cumulative displacements for the same radii were different. As for the URF, the cumulative displacement was far higher for microbubbles compared to fat particles.

Fig.5 shows that the cumulative displacements of gaseous microemboli were of a few hundred microns while those obtained for fat microemboli were of less than ten microns. Note that, as the cumulative displacement obtained for a fat microembolus was less than $10 \mu m$ in the size range under consideration, only cumulative displacements of air microbubbles are considered below. Fig.5 shows that the higher the pressure level, the higher the cumulative displacement. This trend can also seen in Table 3.

Fig.5 also shows that the mass effect, which is expressed as a force resisting the radiation force, has a significant effect on microembolus displacement. This mass effect is clearly described in equation 1 expressing Newton's law. For a fixed size, the microembolus displacement due to the radiation force will be greater for a microbubble (lightweight) than for a microparticle of fat (heavy): the heavier the microembolus the more difficult its displacement. It can thus be assumed that in practice for larger microbubbles and microparticles the microembolus displacement will be less for larger sizes.

\section{Measurable parameters}

Measurable parameters such as $\Delta_{D}, F_{D}(t), F M I(t), P M I(t)$ are presented in Table 2 for different pressure levels and in Table 3 for different velocity values.

As Table 2 shows, the lower the losses, the higher the maximum Doppler frequency, the higher the maximum of the frequency modulation index and the higher the maximum of the position modulation index.

As shown in Table 3, the higher the flow velocity, the lower the transit time, the lower 
the number of pulses, and the lower the cumulative displacement. Indeed, the faster the blood flow, the less often the microbubble is pushed and the lower the cumulative effect. On the other hand, the higher the blood velocity, the higher the Doppler frequency, FMI and PMI. The radial and the longitudinal cumulative displacements were in the order of a few tens of microns and a few tens of millimeters, respectively.

Note also in Table 2 and Table 3 that FMI and PMI were in the order of a few kiloHertz and a few hundred millimeters, respectively.

\section{Acoustic beam and velocity profile}

Fig. 6 shows that the cumulative displacement, Doppler frequency, frequency modulation index and position modulation index were very different for different configurations (plug velocity-flat US beam, plug velocity-Gaussian US beam, laminar velocity-flat US beam, laminar velocity-flat US beam). This suggests that the spatial distribution of the velocity and the pressure level have important roles in the dynamics of displacement.

Maximum $F_{D}, P M I$ and $F M I$ were obtained for plug and flat profiles. $\operatorname{Max}(F M I)$ was about $30 \mathrm{kHz}$ for a gaseous microembolus of $100 \mu \mathrm{m}$ radius whereas for a gaseous microembolus of $2 \mu \mathrm{m}$ the $\max (F M I)$ reached $100 \mathrm{kHz} / \mathrm{s}$. The size of the microembolus impacted considerably on the $F M I$.

\section{Simulated FM signatures}

In order to prove definitively that the URF can explain all the FM signatures reported in the literature, a series of temporal and time-frequency representations of simulated Doppler MES is presented in Fig.7, similar to those obtained by Tortoli et al. (2000) for the three types of FM identified by Smith et al. (1997). The microembolus was located near the upper wall of the artery or near the center of the artery. The velocity profile was laminar, and the size of the microembolus was 100 microns. 
Fig. 7a shows the temporal and time-frequency representations of a fat microembolus.

As the radiation force was very weak, no frequency modulation was observed.

Fig.7c shows the temporal and time-frequency representations of a gaseous microembolus (a gradual modulation (type II) was observed) located near the upper wall of the artery for a low beam-to-flow angle and a laminar velocity profile. The predominant microdisplacement induced by the radiation force in these conditions was mainly longitudinal and involved no rapid changes in the spectral contents (force principally pushed in the flow direction) but rather a gradual increase or decrease. Note that when the beam-to-flow angle was smaller, the transit time was longer and the FMI (or PMI) lower but the measurement of the FMI was easier (or PMI). As Smith et al. (1997) reported that type II frequency modulations were also probably due to formed elements, we conjectured that solid microemboli greater than 100 microns could generate such an FMS.

Figs. $7 \mathrm{~b}$ and $7 \mathrm{~d}$ show the temporal and time-frequency representations of a gaseous microembolus located near the upper wall of the artery, a high beam-to-flow angle and a laminar velocity profile. In these conditions the predominant microdisplacement induced by the radiation force was mainly radial and involved rapid changes in the spectral content (the force principally pushed in the orthogonal direction of the blood flow). Note that the transit time was shorter and the FMI (or PMI) higher the greater the beam-to-flow angle but the measurement of the FMI (or PMI) was more difficult.

The gaseous microembolus in Fig.7d was located near the center of the artery, the angle was high and the velocity profile was laminar. In these conditions, the microbubble accelerated from the upper wall up to the center of the artery and decelerated to go outside the sample volume. The FM resembled an inverted "V" shape.

By comparing the FMI measured from the time-frequency domain of the simulated Doppler signal, $F M I<0.1 \mathrm{kHz}$ for a solid particle and $F M I>20 \mathrm{kHz}$ for a microbubble, we showed that it was possible to discriminate gaseous from solid microemboli. 
To summarize, we showed that microdisplacements measured directly by PMI and indirectly by FMI are far greater for microbubbles than for formed elements. On the other hand, we established that the lower the acoustic losses, the higher the FMI and PMI. We also showed that the most important parameters which affected trajectory modification that could help to explain the variations in FMS were the spatial velocity distribution, the spatial pressure distribution, the beam-to-flow angle and the size of the microbubble.

\section{Discussion and Summary}

This section is divided into two parts, the first contains the discussion of the radiation force and the second the microdisplacements measured by FMI and PMI.

\section{Ultrasound radiation force}

As already demonstrated by Doinikov (1998) for a solid particulate in a non-perfect fluid, we showed that the radiation pressure exerted by the progressive plane wave was no longer proportional to $\left(R_{0}^{6} k^{4}\right)$, as in the model presented by King (1934), but was proportional to $\left(R_{0}^{2} k\right)$. Thus, even though they are small (see Fig.2), the viscous and thermal effects can cause substantial modifications and increased radiation pressure. Note that the predominating loss for radii smaller than ten microns originated from thermal phenomena whereas the predominating loss for radii greater than ten microns originated from acoustic phenomena, indicating that the best descriptive model is the Yosioka and Kawasima (1955) model.

The results illustrated in Fig.3 are very interesting because they show that the URF acting on a gaseous microembolus was far higher than that acting on a fat microembolus. This is explained by the fact that the sudden change in impedance between plasma and the air microbubble was greater than that between plasma and the piece of fat. This result was valid for radii less than $100 \mu \mathrm{m}$, whatever the model used. The results illustrated in 
Fig.3 also show that the URF (Doinikov model) for microembolus sizes ranging from 10 to 100 microns acting on a microbubble was always greater than that acting on a fat microembolus. These results are encouraging for the purpose of discrimination. Indeed, using only Doinikov's theory, it can be seen from the simulations plotted on Fig. 3 that it is possible to discriminate between gaseous and fat microemboli ranging in radius between $1 \mu \mathrm{m}$ and $100 \mu \mathrm{m}$ by setting a detection threshold at $10 \mathrm{nN}$, discriminating in practice between small gaseous microemboli (a few tens of microns) and large solid microemboli (a few hundred microns).

By comparing the different models presented, it can be seen that the asymptotic behavior of the Yosioka and Kawasima (1955) model was similar to that of the Doinikov (1998) model for large radii while for small radii the Yosioka and Kawasima model underestimated the URF: this was due mainly to the thermal and viscous effects. More precisely, as illustrated in Fig.4, the viscous and thermal effects predominated for very small microbubbles compared to the acoustic damping, i.e. when $\left(k R_{0}\right)^{3}<<\delta_{v} / R_{0}<<1$ and $\left(k R_{0}\right)^{3}<<\delta_{t} / R_{0}<<1$. This was confirmed by the viscosity damping formula $\left(\beta_{v i s}=\frac{2 \eta}{\rho R_{0}^{2}}\right)$ which is inversely proportional to the microbubble radius: the smaller the microbubble, the higher the viscous damping. It should also be noted that another consequence of the effects of thermal and viscous damping was that, apart from the resonance $\omega_{0}^{\prime}$, the radiation force given by eqn.(5) was greater than that obtained for a perfect fluid (i.e. the Yosioka and Kawasima model). This is in good agreement with the fact that damping effects reduce the amplitude of the radiation force at the resonance frequency.

Moreover, due to the surface tension, the resonance angular frequency $\omega_{0}$ corresponding to the Yosioka and Kawasima model (surface tension not taken into account in this model) was slightly different from $\omega_{0}^{\prime}$ in the Doinikov model $\left(\omega_{0}^{\prime}>\omega_{0}\right)$.

By comparing the model proposed by Leighton (1994), it could be seen that the asymptotic behaviors for both small and large radii were different from those of the Doinikov model. This was mainly due to the fact that the total damping term $\left(\beta_{t o t}=\delta_{t o t} \omega_{0}^{\prime} / 2=\right.$ 
$\left.0.1 \omega_{0}^{\prime}\right)$ proposed by Leighton (1994) is different from the total damping term proposed by Doinikov (1998) (eqn.(7)) and also to the fact that the resonance angular frequency $\omega_{0}^{\prime}=\omega_{0} \sqrt{1+2 K_{3}}$ proposed by Tortoli et al. (2000) is slightly different from the resonance angular frequency $\omega_{0}^{\prime}=\omega_{0} \sqrt{1-K_{3}}$ proposed by Doinikov (1998). The resonance angular frequency presented by Tortoli et al. (2000) was between $\omega_{0}$ and the $\omega_{0}^{\prime}$ defined by Doinikov (1998).

Note that, three theoretical models of the URF acting on a single microbubble can be recognized with different values of $K, K_{2}$ and $K_{3}$ (see below):

- the Doinikov model (eqn. (5)) where $K_{2}=1-K_{3}$ with $K_{3}=\frac{2 \tilde{\sigma}}{3 R_{0} \tilde{\rho} \tilde{c}^{2}}$;

- the Yosioka and Kawasima (1955) model for (eqn. (5)) where $\beta_{v i s}=\beta_{t h}=0, K=1$, $K_{3}=0$ and $K_{2}=1 ;$

- the Leighton (1994) (p.343), and Dayton et al. (1997) models, (where $K=1$ in eqn. (5)), and Tortoli et al. (2000)'s model (where $K=1, K_{2}=1+2 K_{3}$ and $2 \beta_{\text {tot }}=\delta_{\text {tot }} \omega_{0}$ in eqn. (5). The $\delta_{t o t}$ for a microbubble of 2 microns radius obtained at resonance frequency in water $\left(f_{0}^{\prime}=1.83 \mathrm{MHz}\right)$ is equal to 0.2 (see Leighton (1994)). The latter model provides a radiation force which is almost always greater than that provided by the Doinikov model. Note also that the resonance angular frequency is between $\omega_{0}^{\prime}$ and $\omega_{0}$.

In summary, the URF acting on a microbubble is very different from that acting on a fat particle both in terms of levels and in terms of trends whatever the model. More precisely, whatever the size range studied, the URF acting on a microbubble is far higher than that acting on a fat particle, which is an important point for discrimination purposes.

\section{Displacement}

We discuss here the cumulative displacement of a microbubble, the measurable parameters, parameters which impact on the microembolus trajectory and the different FM 
signatures observed in practice.

470

\section{Cumulative displacement}

The displacement analysis showed that:

- the cumulative displacement of gaseous microemboli (a few hundred microns) was greater than those obtained for fat microemboli (less than ten microns). This deviation by a factor of ten corroborates the above results concerning the radiation force, and is encouraging for discrimination purposes;

- for roughly similar conditions the simulated microbubble displacement (a few hundred microns) was similar to that measured by Palanchon et al. (2005), indicating that the models proposed by Leighton (1994) and adapted by Tortoli et al. (2000) and Doinikov (1996) were good models and probably appropriate;

- the cumulative displacement increased with the pressure level, explained in part by the fact that the URF was proportional to the pressure level $P$.

- the evolution of the cumulative displacement in relation to microembolus size followed the same trends as the radiation force. These results were consistent. However, there were some small differences, mainly explained by the fact that the mass effect was greater for high microembolus sizes, suggesting that as size increases the displacement decreases. This seems to demonstrate that the signature will be large and the displacement will be small for large fat particles, whereas both the signature and the displacement will be large for large bubbles. This hypothesis remains to be confirmed.

\section{Measurable parameters}

It is interesting that all parameter values (summarized in Table 2) were higher for higher pressure levels, except for the transit time and the number of pulses. This phenomenon is readily explained by the fact that the radiation force was stronger and the microembolus velocity was higher when the acoustic pressure was greater (or the displacement per time 
unit). Since the Doppler frequency was proportional to the microembolus velocity, the higher the velocity the higher the Doppler frequency. The same applied for FMI and PMI. Lastly, both the time spent by the microembolus in the sample volume and the number of pulses were reduced by increasing the velocity.

It should be noted that, although the reported displacements were small, they were nonnegligible with regard to a TCD system. Indeed, by assuming a typical system with $8 \mathrm{kHz}$ PRF and 128 point FFT (the time resolution being 128/8000=16 ms and the spectral resolution $8000 / 128=62.5 \mathrm{~Hz}$ ), the lowest detectable FMI would be $62.5 \mathrm{~Hz} / 16 \mathrm{~ms}=3.9 \mathrm{kHz} / \mathrm{s}$. This implies that the lowest detectable FMI corresponds to losses below $10 \mathrm{~dB}$, which seems to correspond to patients with a narrow temporal window. If we consider that the radiation force is indeed the phenomenon which underlies the presence of a high FMI or the presence of a "V" shaped time-frequency, it would be logical to suggest that these phenomena would be observed only in patients with a narrow temporal window.

Moreover, comparing the simulated values to the practical values reported by (Girault et al., 2010) showed that, though the experiment slightly differed from the simulation, the differences between them were small. For example, comparing FMI values extracted from Table 3 for $150 \mathrm{kPa}(0.23<\max (F M I)<2.04 \mathrm{kHz} / \mathrm{s})$ with the measured $F M I$ extracted from Fig.10 in (Girault et al., 2010) with a skull $(0.16<\max (F M I)<2$ $\mathrm{kHz} / \mathrm{s})$ and $F M I$ values extracted from Table 3 for $500 \mathrm{kPa}(3.52<\max (F M I)<19.46$ kHz/s) with the measured FMI extracted from Fig.10 in (Girault et al., 2010) without a skull $(4<F M I<40 \mathrm{kHz} / \mathrm{s})$, it was obvious that these values were of the same order, in part validating the simulation models. We attributed the discrepancy to the fact that the surrounding medium was different (blood/water) and also to the fact that parameters such as microembolus size, fluid velocity, temperature, etc, had not been clearly established or were not known.

To summarize, it is easier to execute practical measurements when the pressure level is high than when the duration of the Doppler signal is low. Furthermore, the thinner the 
temporal bone the easier it is to execute the measurements. Moreover, though conditions differed slightly, the practical values reported in the literature are similar to our simulated values.

\section{Acoustic beam and velocity profile}

The findings extracted from Fig. 6 show clearly that the spatial distributions of both velocity and pressure have important roles in terms of shape and values. The spatial distribution of pressure has a marked role in the shape of the FMS rather than in terms of the Doppler frequency value. In contrast, the spatial distribution of velocity has a marked role in terms of Doppler frequency value rather than in the shape of the FMS. In the case of a pulsatile flow, the shape and the value of the additional acceleration seems to be very complex and unpredictable. Note also that the size of the microbubble seems to be another parameter which affects the microbubble trajectory.

\section{Simulated FM signatures}

Finally, the results illustrated in Fig. 7 show considerable similarities between the in vivo and in vitro experiments reported in the literature (Smith et al. (1997), Girault et al. (2010)) and in our simulations, seeming to confirm that our models are very applicable to this situation.

We also showed that by setting a constant threshold of $1 \mathrm{kHz} / \mathrm{s}$ it was possible to differentiate between microbubbles and solid particles from FMI evaluated from simulated Doppler signals, confirming previously published results (Smith et al. (1997)).

We showed that the beam-to-flow angle, the type and size of the microembolus, the spatial distribution of the velocity and the pressure level all have non-negligible roles in the FMS, demonstrating that it is possible to explain all FMS for the following reasons: 
- For "type I" the radiation is not enough to push the solid particle, and therefore no

FM is present. The microembolus circulates at the same velocity as blood;

- For "type II", involving microbubbles and large micro-particles, the UFR acts in the same direction as the blood flow and thus microemboli are accelerated gradually;

- For "type III" involving only microbubbles:

(1) The first explanation is based on the fact that it is necessary to take into account the pulsatility of flow. Thus, on the basis that the blood flow is time-varying, and that outside the sample volume the microbubble is near the center of the artery during the diastole phase, at this instant in time the microbubble velocity is quasi maximal. When the microbubble enters the sample volume, it moves towards the wall due to the radiation force, and the microbubble decelerates. A few milliseconds later, the microbubble enters the systole phase with the same flow-line as in the diastole phase. The microbubble then accelerates, since the maximum value of the flow accelerates. As the microbubble is pushed towards the artery wall, the microbubble slows down. The form of the FM thus drawn resembles a "V".

(2) For the last explanation, instead of focusing on the velocity profile, it is also possible to explain the "V" shape or the inverted "V" shape by taking into account only the spatial variations present in the ultrasound field. By assuming a plug velocity profile (ensuring no spatial variations in the blood velocity), if the US beam is located in such a way that the principal lobe of the US beam in the sample volume is located near the upper wall and the second lobe is located near the lower wall, then the pressure level in the center of the artery is very low. A "V" shape is then visualized on the TCD for this configuration since the high displacements are near the walls whereas low displacements are in the center. It can be seen that it is possible to image most of the unusual and varied FMS by combining both the velocity and the US beam profiles.

However, it should be noted that detection of a Type III FMS rules out solid emboli and therefore the physician will understand that no dramatic action is required. Detection 
of a non-Type III FMS does not characterize a solid or a gas, because we do not know about emboli $>100$ microns. Further studies are therefore required to provide information regarding the significance of non-Type III FMS signals for physicians.

To summarize, two analytical models of the URF and a numerical model of displacement are proposed. Simulation results, which were in a good agreement with experiments reported in the literature, validated the numerical models, thus confirming that the radiation force should no longer be ignored in the process generating the various shapes of the FM signatures.

The most important parameters which affect and therefore explain most FMS encountered in practice are the spatial distributions of velocity and pressure, the pulsatility of the blood flow, microbubble size and the beam-to-flow angle. Several valid hypotheses are proposed to explain the different types of FMS. Finally, our study confirms that FMI (and PMI) provide valuable information on which discrimination techniques can be based.

\section{Acknowledgements}

This study was supported by the European Union (UMEDS Project) and by the French Government (CASC Project). We wish to thank P. Tortoli for valuable discussions.

\section{References}

Asaki, T. J., Marston, P., 1994. Acoustic radiation force on a bubble driven above resonance. J. Acoust. Soc. Am, 3096-3099.

Benocci, C., Buchlin, J.-M., Michelassi, V., Weinacht, P., 1986. Numerical modelling of gas-droplet flows for industrial applications. presented at the 3rd Int. Con. Computationnal Methods Expe. Measure., Porto Carras, Greece,. 
Cowe, J., Grittins, J., Naylor, A., Evans, D., 2002. Rf signals provide additional information on embolic events recorded during tcd monitoring. Ultrasound in medecine and biology 31, 613-623.

Dayton, P. A., Morgan, K. E., Klibanov, A. L., Brandenburger, G., Nightingale, K. R., Ferrara, K. W., 1997. A preliminary evaluation of the effects of primary and secondary radiation forces on acoustic contrast agents. IEEE Trans. Ultrason., Ferroelect., Freq. Contr. 44, $1264 ? 1277$.

Devuyst, G., Darbellay, G., Vesin, J.-M., Kemeny, V., Ritter, M., Droste, D., Moline, C., Serena, J., Sztajzel, R., Ruchat, P., ang G. Dietler, C. L., Ringelstein, E., 2001. Automatic classification of hits into artifacts or solid or gaseous emboli by a wavelet representation combined with dual gated tcd. Ultrasound in medecine and biology 27, 2803-2809.

Devuyst, G., Vesin, J.-M., Despland, P.-A., Bogousslavsky, J., 2000. The matching pusruit method: a new method of characterizing micro-emboli signals ? Ultrasound in medecine and biology 26, 1051-1056.

Doinikov, A., 1996. On the radiation pressure on a small spheres. J. Acoust. Soc. Am. 100, 1231-1233.

Doinikov, A., 1997a. Acoustic radiation force on a spherical particle in a viscous heatconducting fluid. i. general formula. J. Acoust. Soc. Am. 101, 713-721.

Doinikov, A., 1997b. Acoustic radiation force on a spherical particle in a viscous heatconducting fluid. ii. force on a rigid sphere. J. Acoust. Soc. Am. 101, 722-730.

Doinikov, A., 1997c. Acoustic radiation force on a spherical particle in a viscous heatconducting fluid. iii. force on a liquid drop. J. Acoust. Soc. Am. 101, 731-740.

Doinikov, A., 1998. Acoustic radiation force on bubble: viscous and thermal effect. J. Acoust. Soc. Am. 103, 143-147.

Georgiadis, D., Mackay, T., Kelman, A., Grosset, D., Wheatley, D., Lees, D., 1994. Differentiation between gaseous and formed embolic materials in vivo. application in prosthetic heart valve patients. Stroke 25, 1559-1563.

Girault, J.-M., Kouame, D., Guidi, F., Menigot, S., Souchon, G., Tranquart, F., 2010. 
Embolus discrimination by means of ultrasound radiation force part ii: Analysis of the frequency modulation by means of in vitro experiments. Ultrasound in medecine and biology XX, XX-XX.

Guidi, F., Boni, E., Tortoli, P., 2003. Acoustic method for real-time visualization of microbubble movements and rupture. IEEE Ultrasonics symposium, 1183-1186.

King, L., 1934. On the acoustic radiation pressure on spheres. Proc. Roy. Soc. A. 147, $212-240$.

Leighton, T. G., 1994. The acoustic bubble. New York: AcademicPress.

Markus, H., Brown, M., 1993. Differentiation between different pathological cerebral embolic materials using transcranial doppler in a in vitro model. Stroke 24, 1-5.

Mess, W., Willigiers, J., Ledoux, L., Ackerstaff, R., Hoeks, A., 2002. Microembolic signal description: a reappraisal based on a custumized digital postprocessing system. Ultrasound in medecine and biology 28, 1147-1455.

Palanchon, P., Tortoli, P., Bouakaz, A., Versluis, M., DeJong, N., 2005. Optical observation of acoustical radiation force effects on individual air bubbles. IEEE Trans. Ultrason., Ferroelect., Freq. Contr., 104-110.

Prosperetti, A., 1977. Thermal effects and damping mechanisms in the forced radial oscillations of gas bubbles in liquids. J. Acoust. Soc. Am., 17-27.

Rusell, D., Brucher, R., 2002. Online automatic discrimination between solid and gaseous cerebral microemboli with the first multifrequency transcranial doppler. Stroke 33, $1975-1980$.

Rusell, D., Madden, K., Clark, W., Sanset, P., Zivin, J., 1991. Detection of arterial emboli using doppler ultrasound in rabbits. Stroke 22, 253-258.

Smith, J., Evans, D., Bell, P., Naylor, R., 1998. A comparison of four methods for distinguishing doppler signals from gaseous and particulate emboli. Ultrasound in medecine and biology 29, 1133-1138.

Smith, J., Evans, D., Naylor, R., 1997. Analysis of the frequency modulation present in doppler ultrasound signal may allow differentiation between partiulate and gaseous cerebral emboli. Ultrasound in medecine and biology 23, 727-734. 
Souchon, G., Girault, J.-M., Biard, M., Kouamé, D., Tranquart, F., 2005. Gaseous and solid emboli differentiation using radiation force. IEEE Ultrasonics symposium, Rotterdam, 2070-2073.

Tortoli, P., Pratesi, M., Michelasi, V., 2000. Doppler spectra from contrast agents crossing an ultrasound field. IEEE Trans. Ultrason., Ferroelect., Freq. Contr. 47, 716-725.

Yosioka, K., Kawasima, Y., 1955. Acoustic radiation pressure on a compressible sphere. Acustica 5, 167-173. 
$2 \quad F_{D}, F M I$ and $P M I$ for a gaseous microembolus circulating in plasma, $I_{\text {spta }}=720 \mathrm{~mW} / \mathrm{cm}^{2}, P R F=8 \mathrm{kHz}, N=40, L=23 \mathbf{m m}$, $V=1 \mathrm{~m} / \mathrm{s}, \theta=5$ degrees, $R_{0}=100 \mu \mathrm{m}$, uniform (Plug) or laminar velocity profiles and uniform (Flat) or Gaussian US beam profiles.

3 Maximum FMI and maximum PMI for gaseous microemboli $(100 \mu \mathbf{m})$ circulating in plasma $(\eta=4 \mathbf{m P a} . \mathbf{s}), P R F=8 \mathrm{kHz}$, $f=2 \mathrm{MHz}, N=40, L=23 \mathrm{~mm}, \theta=5$ degrees for a laminar velocity profile and for a gaussian US beam. We assumed a gaseous microembolus with a radius of $R_{0}=100 \mu \mathrm{m}$ circulating in blood at a maximum velocity of $V=0.5 \mathrm{~m} / \mathrm{s}$ in an artery of $5 \mathrm{~mm}$. The initial position of the microembolus in the artery was $4.5 \mathrm{~mm}$. 
$2 \quad k^{3} R_{0}^{3}, \delta_{v} / R_{0}, \delta_{t} / R_{0}$ weighting factors for a fat microembolus embedded in blood. Viscous and thermal effects in relation to radius. These combined effects (viscous and thermal) predominated for microemboli below $30 \mu \mathrm{m}$. The pressure level was set at $P=500 \mathrm{kPa}$.

3 Discrimination between gaseous and solid microemboli circulating in plasma ( $\eta=4$ mPa.s) using simplified Doinikov (1996),Doinikov (1998), Yosioka and Kawasima (1955) and Dayton et al. (1997) models. These curves show that viscous and thermal effects predominate below $30 \mu \mathrm{m}$. Above $30 \mu \mathrm{m}$ the simplified Doinikov (1998) model was similar to the Yosioka and Kawasima (1955) model. Note that the model proposed by Dayton et al. (1997) did not give the same asymptotic value for high frequencies. Note also that the radiation force acting on a microbubble of $2 \mu \mathrm{m}$ is equivalent to that acting on a microbubble of $20 \mu \mathrm{m}$.

4 Weighting factors of eqn.(10) and damping coefficients of eqn.(7) for a gaseous microembolus immersed in blood. Viscous and thermal effects in relation to radius. These combined effects predominated for microemboli below $30 \mu \mathrm{m}$. 
5 Cumulative displacement $\Delta_{D}^{*}$ of a gaseous microembolus in blood $(\eta=4 \mathrm{mPa} . \mathrm{s})$. Microembolus diameters ranged from $1 \mu \mathrm{m}$ to $100 \mu \mathrm{m}$ (in order to verify the validity of the physical model). $P F R=8 \mathrm{kHz}$ (commonly used PRF are 1,5,6,8,10 kHz), $P a=500-150 \mathrm{kPa}$, $f=2 \mathrm{MHz}, V=0.1 \mathrm{~m} / \mathrm{s}, N=20$, laminar profile, gaussian US beam, simplified Doinikov model. The duration of the pulse train was $10 \mu \mathrm{s}$ $(4 \mu \mathrm{m}$, (Mess et al., 2002) $<10 \mu \mathrm{m}<13 \mu \mathrm{m}$ (Cowe et al., 2002)). The microembolus immersed in blood moved in a section of artery of $5 \mathrm{~mm}$ and the insonification angle was 5 degrees. Note that the US beam width revealing the microembolus was: $L=23 \mathrm{~mm}$. The physical parameters for the medium studied are shown in Table 1. The initial position of the microembolus was set at $4.5 \mathrm{~mm}$, i.e. near the upper wall of the tube ( 5 $\mathrm{mm})$. The microemboli were spherical.

6 Cumulative displacement, Doppler frequency, FMI and PMI for different fluid velocities in the case of a gaseous microembolus $\left(R_{0}=100 \mu \mathrm{m}\right)$ circulating in plasma. $V=0.5 \mathrm{~m} / \mathrm{s}, L=23 \mathrm{~mm}, P R F=8 \mathrm{kHz}, f=2$ $\mathrm{MHz}, N=20, P=500 \mathrm{kPa}$.

7 Time frequency representation of a simulated microembolic Doppler signal. a) As $F M I<0.1 \mathrm{kHz}$, we concluded that there was no FM modulation (fat particle). c) Gradual modulation observed over most of the signal duration (microbubble near the upper artery wall), $F M I>20$ kHz. b) Rapid change (reversed "V" shape) in the FM on a small percentage of the signal duration (microbubble near the artery center). The reversed "V" shape, which seems to be representative of a gaseous microembolic signature, is similar to that reported by Smith et al. (1997) and Girault et al. (2010). d) Rapid change in the FM on a small percentage of the signal duration (microbubble near the upper artery wall). FMI evaluated in the accelerating phase was $\Delta_{f} / \Delta_{t}=500 / 0.016=31 \mathrm{kHz} / \mathrm{s}$. 
Table 1

\section{Physical parameters}

\begin{tabular}{|c|c|c|c|c|}
\hline \hline \multirow{2}{*}{\begin{tabular}{c} 
parameters/medium \\
\cline { 2 - 5 }
\end{tabular}} & \multicolumn{2}{|c|}{ host fluid } & \multicolumn{2}{c|}{ microembolus $(\sim)$} \\
\cline { 2 - 5 } & water & plasma & fat & air \\
\hline \hline$c$-speed of sound $(\mathrm{m} / \mathrm{s})$ & 1000 & 1055 & 952 & 1.1 \\
\hline$\eta$ - viscosity $(\mathrm{mPa} . \mathrm{s})$ & 1 & 4 & 40 & 0.018 \\
\hline$\chi$ - thermal diffusivity $(\mu \mathrm{m} / \mathrm{s} / \mathrm{s})$ & 0.10 & 0.11 & 0.20 & 28 \\
\hline$\kappa$ - thermal conductivity $(\mathrm{J} / \mathrm{smK})$ & 0.58 & - & - & 0.034 \\
\hline$\nu$ - specific heat ratio & 1 & - & - & 1.4 \\
\hline
\end{tabular}

Note that the superficial tension of the air microbubble was $\tilde{\sigma}=72.10^{-3} \mathrm{~N} / \mathrm{m}$ and

$$
\tilde{p}_{0}=100 \mathrm{kPa} \text {. }
$$

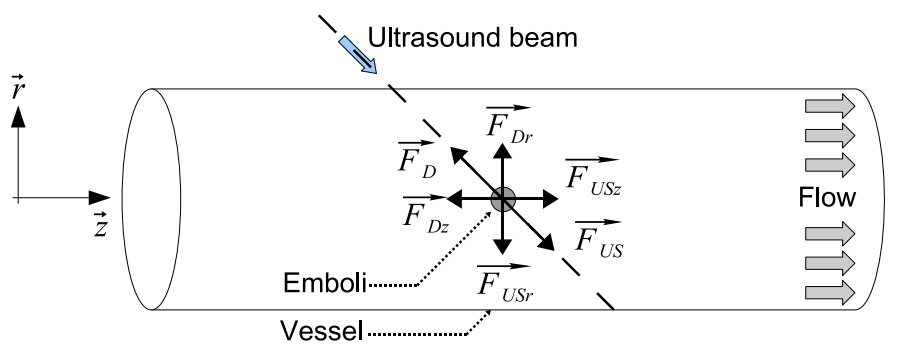

Fig. 1. Forces acting on a microembolus in a fluid flow. 
Table 2

$F_{D}, F M I$ and $P M I$ for a gaseous microembolus circulating in plasma, $I_{s p t a}=$ $720 \mathrm{~mW} / \mathrm{cm}^{2}, P R F=8 \mathrm{kHz}, N=40, L=23 \mathrm{~mm}, V=1 \mathrm{~m} / \mathrm{s}, \theta=5$ degrees, $R_{0}=100$ $\mu \mathrm{m}$, uniform (Plug) or laminar velocity profiles and uniform (Flat) or Gaussian US beam profiles.

\begin{tabular}{|c|c|c|c|c|c|c|c|c|c|c|}
\hline Profiles & \multicolumn{9}{|c|}{ Plug, Flat } & \multicolumn{5}{c|}{ Laminar, Gaussian } \\
\hline loss $(\mathrm{dB})$ & 20 & 15 & 10 & 5 & 0 & 20 & 15 & 10 & 5 & 0 \\
\hline$P(\mathrm{kPa})$ & 38 & 67 & 120 & 210 & 380 & 38 & 67 & 120 & 210 & 380 \\
\hline $\max \left(F_{D}\right)(\mathrm{kHz})$ & 1.87 & 1.88 & 1.89 & 1.91 & 1.95 & 0.68 & 0.68 & 0.69 & 0.71 & 0.76 \\
\hline $\max (F M I)(\mathrm{kHz} / \mathrm{s})$ & 0.62 & 1.92 & 5.84 & 16.8 & 43.2 & 0.14 & 0.43 & 1.32 & 4.00 & 11.32 \\
\hline $\max (P M I)(\mathrm{mm} / \mathrm{s})$ & 981 & 982 & 984 & 993 & 1015 & 353 & 353 & 355 & 360 & 378 \\
\hline
\end{tabular}

To take into account the fact that the pressure level in the brain varies according to the patient, several levels of loss were simulated $(0,5,10,15,20 \mathrm{~dB})$. Different pressure values are proposed to cover the situations most commonly encountered in practice, corresponding to different types of loss such as reflections, diffraction effects and attenuation. To predict the losses encountered in in vivo conditions, the brain attenuation must be taken into account (attenuation coefficient $0.5 \mathrm{~dB} / \mathrm{cm} / \mathrm{MHz}$ ). In this case the corresponding loss at $5 \mathrm{~cm}$ for an emitting frequency of $2 \mathrm{MHz}$ would be $5 \mathrm{~dB}(0.5 \times 5 \times 2)$, and the total loss would be $15 \mathrm{~dB}$. 
Table 3

Maximum FMI and maximum PMI for gaseous microemboli (100 $\mu$ m) circulating in plasma $(\eta=4 \mathrm{mPa} . \mathbf{s}), P R F=8 \mathrm{kHz}, f=2 \mathrm{MHz}, N=40, L=23 \mathbf{m m}, \theta=5$ degrees for a laminar velocity profile and for a gaussian US beam. We assumed a gaseous microembolus with a radius of $R_{0}=100 \mu \mathrm{m}$ circulating in blood at a maximum velocity of $V=0.5 \mathrm{~m} / \mathrm{s}$ in an artery of $5 \mathrm{~mm}$. The initial position of the microembolus in the artery was $4.5 \mathrm{~mm}$.

\begin{tabular}{|c|c|c|c|c|c|c|c|c|}
\hline$P(k P a)$ & \multicolumn{5}{|c|}{150} & \multicolumn{3}{c|}{500} \\
\hline$V_{\text {max }}(\mathrm{m} / \mathrm{s})$ & 0.1 & 0.5 & 0.8 & 1 & 0.1 & 0.5 & 0.8 & 1 \\
\hline$\Delta_{D}^{*}(\mathrm{~mm})$ & 22.94 & 22.95 & 22.96 & 22.98 & 22.95 & 22.96 & 22.97 & 22.98 \\
\hline$\Delta_{r}(\mathrm{~mm})$ & 0.05 & 0.01 & 0.007 & 0.005 & 0.27 & 0.09 & 0.07 & 0.05 \\
\hline$\Delta_{z} *(\mathrm{~mm})$ & 22.94 & 22.95 & 22.96 & 22.98 & 22.95 & 22.96 & 22.97 & 22.98 \\
\hline max $(F M I)(\mathrm{kHz} / \mathrm{s})$ & 0.23 & 1.05 & 1.65 & 2.04 & 3.52 & 10.76 & 16.02 & 19.46 \\
\hline $\max (P M I)(\mathrm{mm} / \mathrm{s})$ & 41 & 180 & 286 & 356 & 93 & 233 & 335 & 403 \\
\hline$t_{t}(\mathrm{~ms})$ & 596 & 126 & 79 & 63 & 470 & 113 & 73 & 59 \\
\hline$N_{\text {pulse }}$ & 1887 & 1004 & 631 & 506 & 1358 & 900 & 588 & 477 \\
\hline \hline
\end{tabular}

If the blood is replaced by water, then for $P=500 \mathrm{kPa}, V_{\max }=0.1 \mathrm{~m} / \mathrm{s}$, and thus the cumulative displacement is $\Delta_{D}^{*}=22.95 \mathrm{~mm}, \Delta_{r}=0.52 \mathrm{~mm}, \max (F M I)=13.2 \mathrm{kHz} / \mathrm{s}$, $\max (P M I)=170 \mathrm{~mm} / \mathrm{s}, t_{t}=394 \mathrm{~ms}$ and $N_{\text {pulse }}=3150$. These values are of the same order as those obtained for blood, and because the viscosity is lower, the microbubble crosses the sample volume more easily and thus more quickly. Note that for other values of $\operatorname{PRF}(1,5,6$ $\mathrm{kHz})$ and $N(8,10,20,30,40)$, the simulation results were very close. 


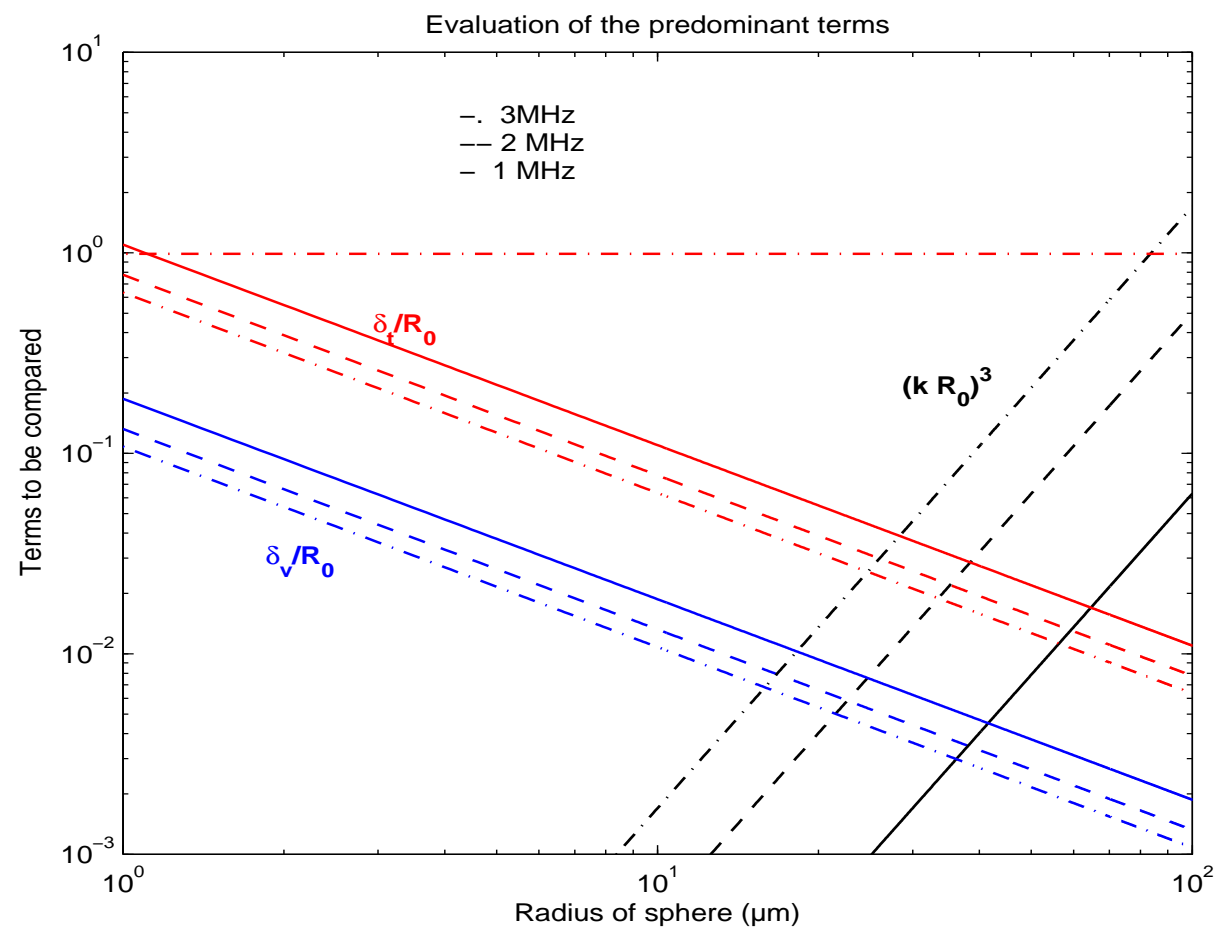

Fig. 2. $k^{3} R_{0}^{3}, \delta_{v} / R_{0}, \delta_{t} / R_{0}$ weighting factors for a fat microembolus embedded in blood. Viscous and thermal effects in relation to radius. These combined effects (viscous and thermal) predominated for microemboli below $30 \mu \mathrm{m}$. The pressure level was set at $P=500 \mathrm{kPa}$. 


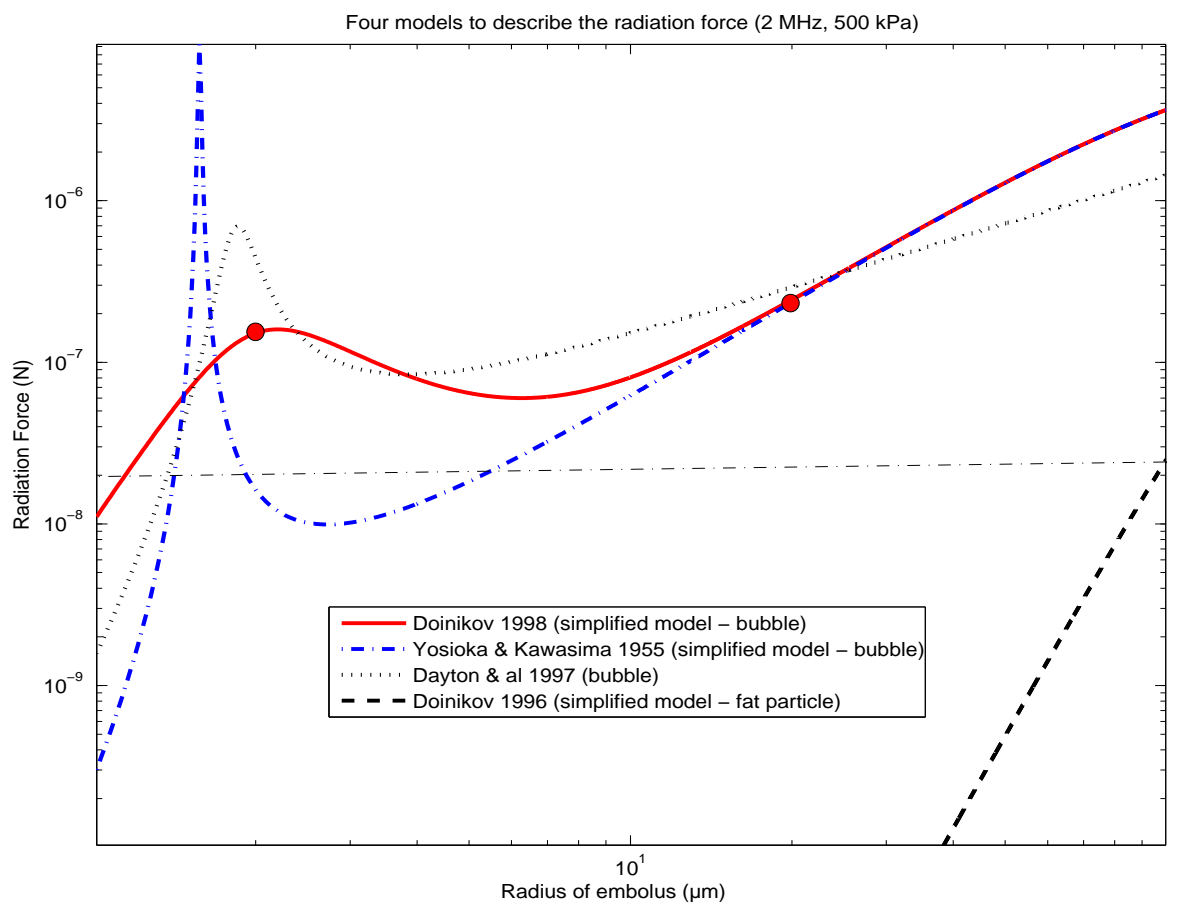

Fig. 3. Discrimination between gaseous and solid microemboli circulating in plasma $(\eta=4$ mPa.s) using simplified Doinikov (1996),Doinikov (1998), Yosioka and Kawasima (1955) and Dayton et al. (1997) models. These curves show that viscous and thermal effects predominate below $30 \mu \mathrm{m}$. Above $30 \mu \mathrm{m}$ the simplified Doinikov (1998) model was similar to the Yosioka and Kawasima (1955) model. Note that the model proposed by Dayton et al. (1997) did not give the same asymptotic value for high frequencies. Note also that the radiation force acting on a microbubble of $2 \mu \mathrm{m}$ is equivalent to that acting on a microbubble of $20 \mu \mathrm{m}$. 

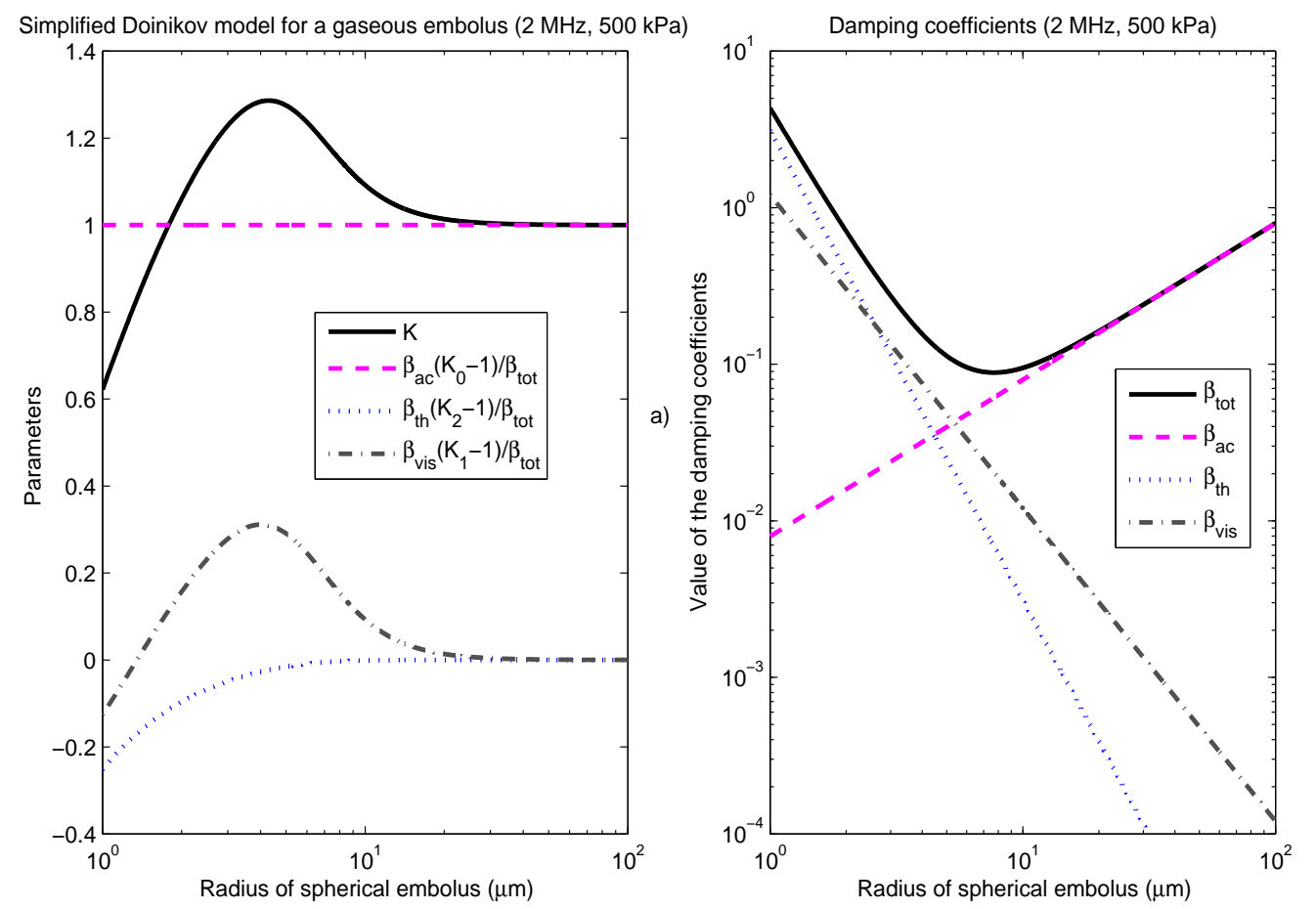

Fig. 4. Weighting factors of eqn.(10) and damping coefficients of eqn.(7) for a gaseous microembolus immersed in blood. Viscous and thermal effects in relation to radius. These combined effects predominated for microemboli below $30 \mu \mathrm{m}$. 


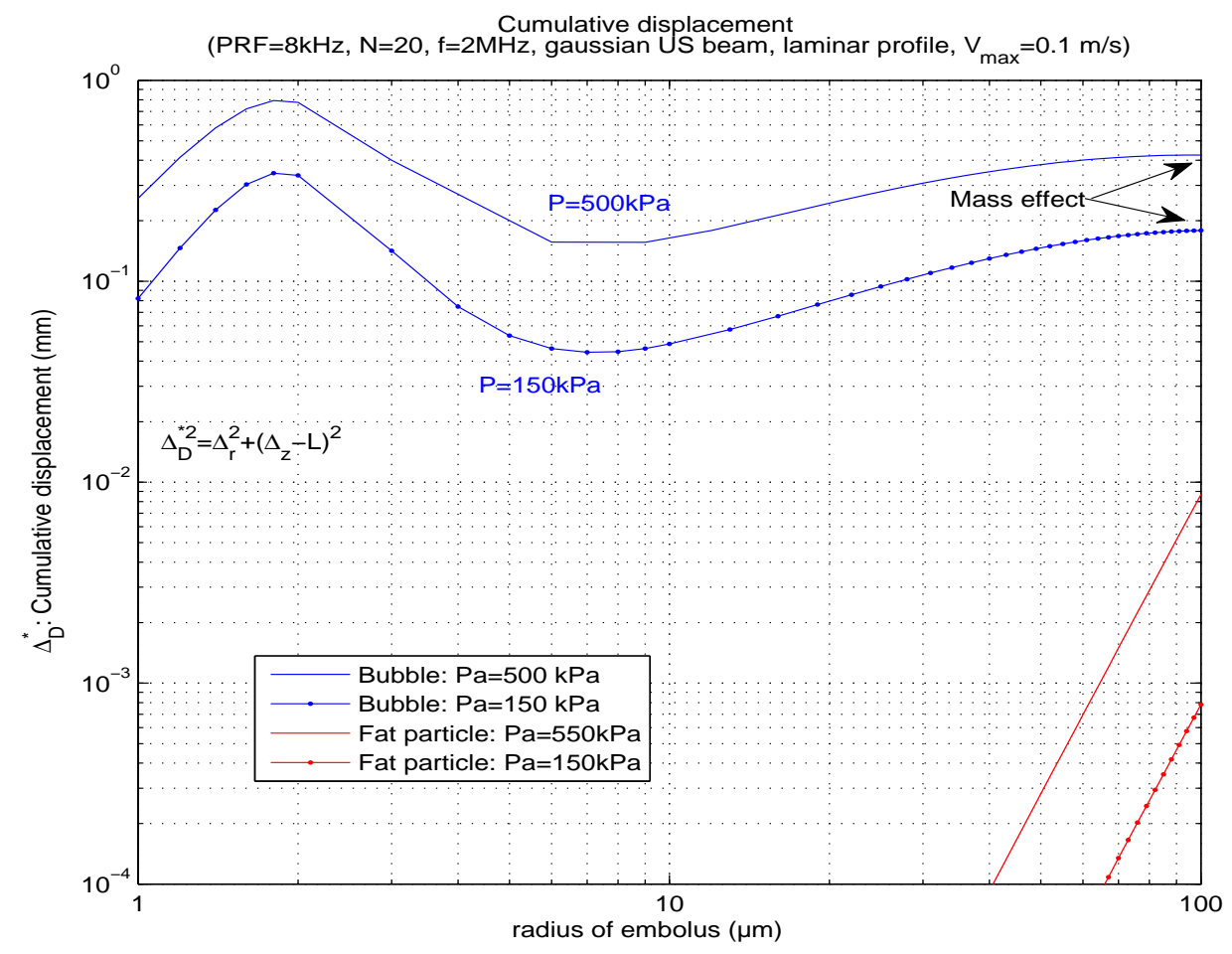

Fig. 5. Cumulative displacement $\Delta_{D}^{*}$ of a gaseous microembolus in blood $(\eta=4 \mathrm{mPa} . \mathrm{s})$. Microembolus diameters ranged from $1 \mu \mathrm{m}$ to $100 \mu \mathrm{m}$ (in order to verify the validity of the physical model). $P F R=8 \mathrm{kHz}$ (commonly used PRF are 1,5,6,8,10kHz), $P a=500-150 \mathrm{kPa}, f=2$ MHz, $V=0.1 \mathrm{~m} / \mathrm{s}, N=20$, laminar profile, gaussian US beam, simplified Doinikov model. The duration of the pulse train was $10 \mu s(4 \mu \mathrm{m}$, (Mess et al., 2002) $<10 \mu \mathrm{m}<13 \mu \mathrm{m}$ (Cowe et al., 2002)). The microembolus immersed in blood moved in a section of artery of $5 \mathrm{~mm}$ and the insonification angle was 5 degrees. Note that the US beam width revealing the microembolus was: $L=23 \mathrm{~mm}$. The physical parameters for the medium studied are shown in Table 1 . The initial position of the microembolus was set at $4.5 \mathrm{~mm}$, i.e. near the upper wall of the tube (5 $\mathrm{mm})$. The microemboli were spherical. 

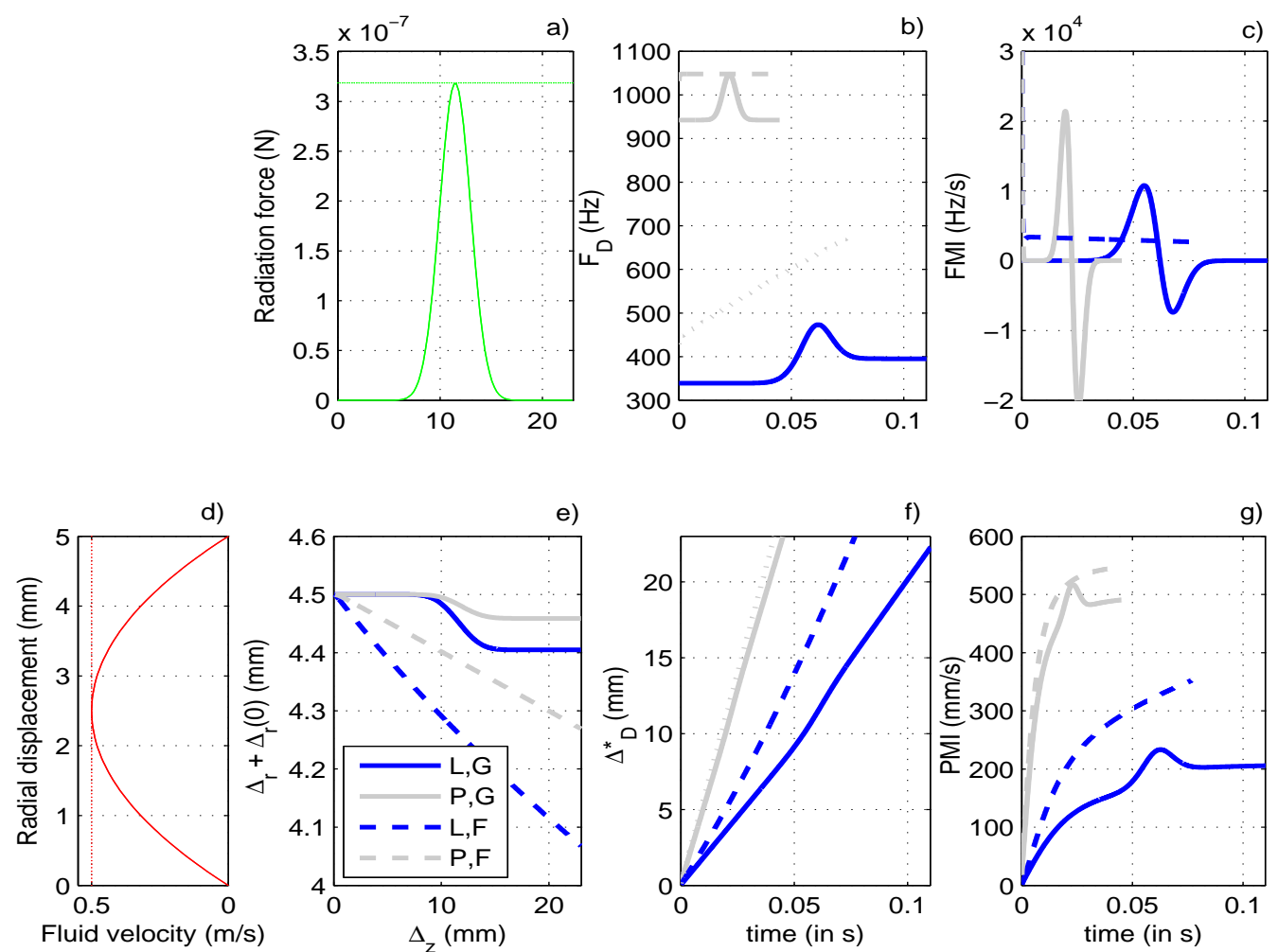

Fig. 6. Cumulative displacement, Doppler frequency, FMI and PMI for different fluid velocities in the case of a gaseous microembolus $\left(R_{0}=100 \mu \mathrm{m}\right)$ circulating in plasma. $V=0.5 \mathrm{~m} / \mathrm{s}$, $L=23 \mathrm{~mm}, P R F=8 \mathrm{kHz}, f=2 \mathrm{MHz}, N=20, P=500 \mathrm{kPa}$. 

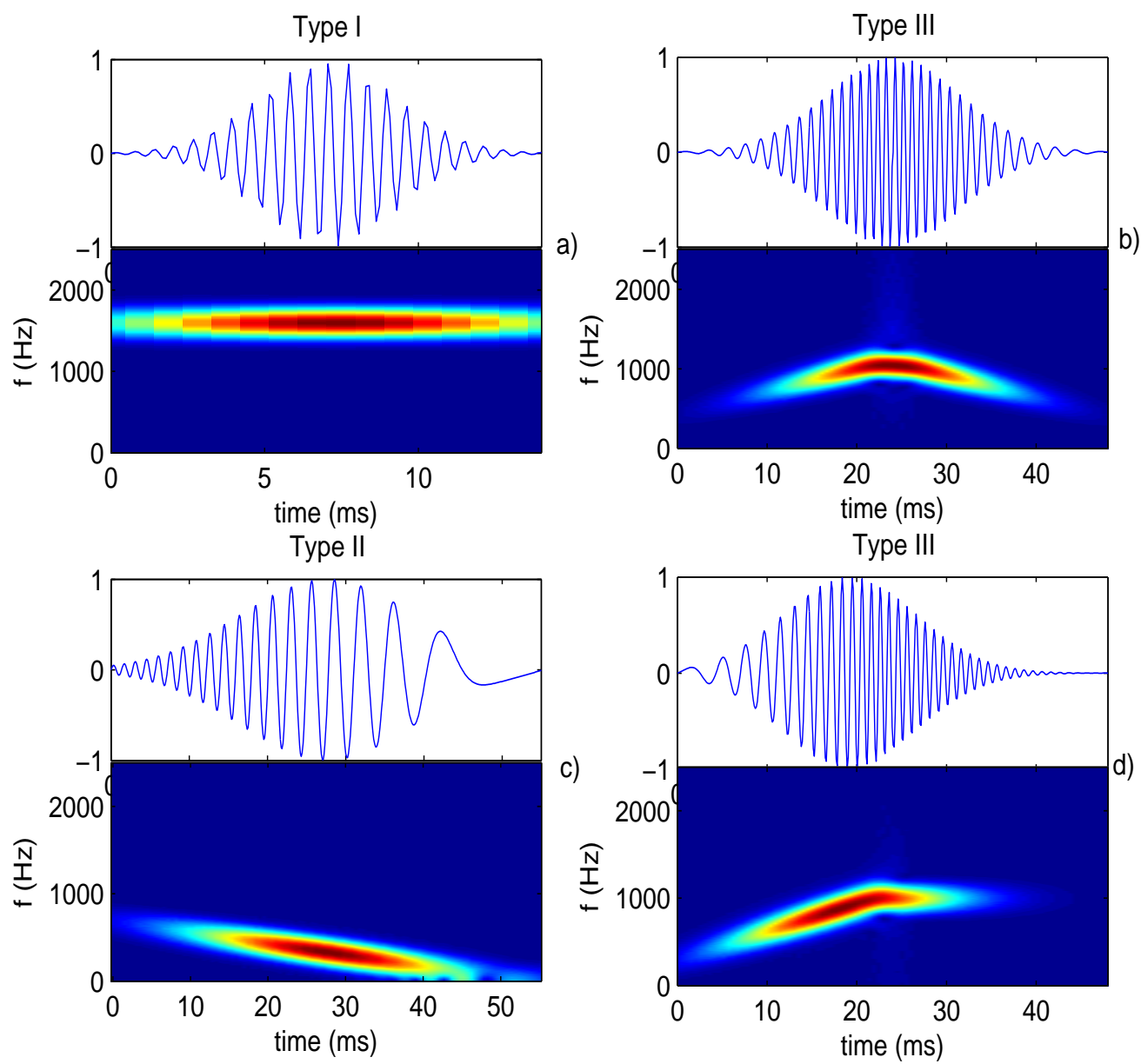

Fig. 7. Time frequency representation of a simulated microembolic Doppler signal. a) As $F M I<0.1 \mathrm{kHz}$, we concluded that there was no FM modulation (fat particle). c) Gradual modulation observed over most of the signal duration (microbubble near the upper artery wall), $F M I>20 \mathrm{kHz} . \mathrm{b}$ ) Rapid change (reversed "V" shape) in the FM on a small percentage of the signal duration (microbubble near the artery center). The reversed "V" shape, which seems to be representative of a gaseous microembolic signature, is similar to that reported by Smith et al. (1997) and Girault et al. (2010). d) Rapid change in the FM on a small percentage of the signal duration (microbubble near the upper artery wall). FMI evaluated in the accelerating phase was $\Delta_{f} / \Delta_{t}=500 / 0.016=31 \mathrm{kHz} / \mathrm{s}$. 